\title{
LA REMUNERACIÓN VARIABLE POR DESEMPEÑO EN EL SECTOR PÚBLICO: ESTUDIO DE LAS CAUSAS DE SU FRACASO Y REPERCUSIONES PARA EL ESTADO BRASILEÑO
}

\author{
Luis Otávio Milagres de Assis \\ otavioassis@yahoo.com.br \\ Fundação Getúlio Vargas - EAESP-FGV, São Paulo, Brasil \\ Mário Teixeira Reis Neto \\ reisnetomario@fumec.br \\ Universidade Fumec, Belo Horizonte, Brasil
}

La implementación de sistemas de remuneración variable por desempeño en la administración pública brasileña ha experimentando un significativo crecimiento en los últimos tiempos, sin embargo el optimismo de los gestores no se basa en la teoría. La literatura muestra que los sistemas de remuneración variable en el sector público en el extranjero fracasan o presentan un éxito limitado. Este artículo tiene como objetivo estudiar las experiencias de implementación de sistemas de incentivos en los gobiernos, analizar las causas de los fracasos ocurridos e identificar aquellas prácticas con posibilidades de llevar al sistema de remuneración variable a generar buenos resultados dentro de la administración pública brasileña. Para ello, se ha investigado la efectividad de los diferentes modelos y estrategias de implementación de los sistemas de incentivos, los aspectos relevantes de la cultura nacional, los argumentos basados en la teoría agente/principal y las interferencias del factor psicológico en la remuneración variable. Para finalizar, se presentan seis prácticas que pueden mejorar los resultados de los sistemas de remuneración variable en el sector público brasileño: la elaboración de una agenda estratégica para toda la organización; el establecimiento de resultados cuantitativos, preferencialmente productos/ servicios, como base de la premiación; la estructuración del modelo en torno a los equipos y no a los individuos; el uso de una unidad de inteligencia contra el gaming; la realización de un proceso formal y periódico de contratación de metas y la creación de sistemas de incentivos particulares en función del tipo de tareas.

Palabras clave: Remuneración variable, incentivos, evaluación del desempeño, sector público. 


\section{PERFORMANCE-RELATED PAY IN THE PUBLIC SECTOR: INVESTIGATION OF FAILURE REASONS AND IMPLICATIONS FOR THE BRAZILIAN STATE}

The installation of performance-related pay at the Brazilian public administration is growing recently, but the optimism of public managers is not grounded on any theory. The literature shows that performancerelated pay at the public sector abroad have failed or obtained a much limited success. This paper has as its objective to study the experiences in installing incentive systems at governments, to analyze the causes of the failures occurred and to identify practices which will have more chances of conducting a variable payment system to generate good results in Brazil's public service. In order to do so, it was investigated the effectiveness of different models and installation strategies for incentive systems, the relevant aspects of national culture, the arguments based on the agent $\mathrm{x}$ principal theory and the interferences of the psychological issue in variable payment. Finally, six practices are presented which may contribute to improve the results of performance related pay at Brazil's public sector, as follows: the construction of a strategic schedule for all the organization; the establishment of quantitative results, preferentially outputs, as basis for the premium; the scheme's modeling around teams rather than around individuals; the use of an intelligence unit against gaming; the performance of a formal and periodic goal contracting process; and the creation of differentiated incentive systems based on the task type. Keywords: performance-related pay, incentive, performance evaluation, public sector. 


\section{INTRODUCCIÓN}

La implementación de los sistemas de remuneración variable (performance-related pay o PRP) en el sector público está marcada por dos momentos: La experiencia del gobierno federal post FHC (Fernando Henrique Cardoso) y la reciente expansión de estas iniciativas dentro de las administraciones públicas brasileñas.

A finales de la década de 1990, el gobierno federal generalizó la implementación de los sistemas de remuneración variable en forma de gratificaciones por desempeńo como un complemento a los sueldos mensuales de la administración pública federal (Pacheco; 2009). En el período 1995-2002, se crearon varias gratificaciones por desempeño. Además de facilitar la concesión de aumentos particulares, la gratificación permitió desvincular la remuneración del personal en activo del que no lo era, y así conceder mayores aumentos a los primeros. Sin embargo, en el gobierno FHC, algunas gratificaciones se extendieron entre el personal no en activo, debido a la presión legal (Pacheco, 2010). En el segundo gobierno de FHC, se produjo una generalización de la remuneración variable por desempeño, pero no vino acompañada de medidas que subsidiasen el sistema, como la contratación de resultados.

En el gobierno de Lula, los valores variables aumentaron de manera significativa, pasando a representar, en muchos casos, valores entre el $22 \%$ y $94 \%$ de las tablas salariales. Rápidamente, las presiones corporativas hicieron que la parte variable fuera íntegramente incorporada al sueldo fijo. Incluso, según Pacheco, el gobierno de Lula adoptó como política la extensión de todas las gratificaciones entre el personal no activo. En Brasil, la mayor parte de las gratificaciones "variables" tienden a ser, en verdad, el prenuncio de un simple aumento salarial. Gran parte de estas gratificaciones acabó, simplemente, siendo incorporada al sueldo, evidenciando un pronóstico sombrío para las experiencias de remuneración variable en el sector público.

Recientemente, concretamente desde el 2008, Brasil parece estar viviendo una nueva ola de implementación de incentivos. Los programas de remuneración variable, generalmente en formato de bonos periódicos semestrales o anuales, se implantaron en diversos gobiernos estatales. Minas Gerais es el estado donde esta iniciativa está más extendida, alcanzando en el 2008, a más del $90 \%$ de los 
funcionarios del ejecutivo del Estado. Según Reis Neto y Assis (2010), de 2004 a 2009 fueron distribuidos 905 millones de reales a título de premiación por productividad. São Paulo implantó el PRP, en el 2009, en las áreas de educación, hacienda y planificación y distribuyó más de 600 millones de reales en premiación ${ }^{1}$. Pernambuco distribuyó entre los funcionarios de educación, en el 2009, un total de 29 millones de reales en función del cumplimiento de las metas definidas para cada escuela $^{2}$. A nivel federal, se aprobó la Ley 12.155 de 23/12/2009, que repartió entre los funcionarios del Departamento Nacional de Infraestructuras de Transportes (DNIT) bonos de 3 mil a 28 mil reales (dependiendo del cargo) en función del cumplimiento de las metas del Programa de Aceleración del Crecimiento (PAC). En Rio de Janeiro, en el 2010, los policías civiles y militares fueron premiados en función de las metas de reducción de la criminalidad, habiendo sido repartidos 6 millones de reales dentro del estado hasta la fecha. ${ }^{3}$

Parece existir un optimismo generalizado entre los gestores públicos en cuanto al potencial de estas iniciativas, pero este optimismo no se basa en la teoría. La literatura muestra que los sistemas de remuneración variable en el sector público fracasan o presentan un éxito limitado (OCDE, 2005; Perry, Engbergs y Jun, 2009; Bowman, 2010; Weibel, Rost y Osterloh 2010). En algunas situaciones, el modelo genera resultados positivos pero, en otros, fracasa en su objetivo, el de motivar al personal y puede hasta generar efectos contrarios.

La experiencia en el gobierno federal brasileño ya muestra una señal de alerta. El uso del PRP como un instrumento de legitimación de los simples aumentos salariales ilustra, de forma contundente, el hecho de que las características culturales brasileñas influyen negativamente en la implementación de los sistemas de evaluación e incentivos, según argumenta Barbosa (1996). Puesto que la remuneración variable en Brasil es bastante reciente, todavía no contamos con trabajos académicos que analicen la implementación de estos modelos ${ }^{4}$.

1 De acuerdo con datos publicados en el Informe de Gestión 2007-2010, Secretaría de Gestión Pública-SP.

2 Diário de Pernambuco, 29/05/2009.

3 Informaciones de la página Web oficial de la Secretaría de Seguridad Pública, acceso 19/06/2010.

4 Un reciente estudio ha mostrado resultados razonablemente positivos en Minas Gerais. Una encuesta (survey) realizada con 339 funcionarios de la Secretaría de Salud señaló que el 56\% de los funcionarios creían que la calidad de su trabajo mejoró con los incentivos dados por la remuneración variable, mientras el $19 \%$ no lo creía así. Sin embargo, sólo se estudió una 
En este escenario, se entienden mejor las razones por las que los sistemas de remuneración variable están presentando resultados contrarios a los deseados en otros países. Este estudio también se justifica en la medida en que la reciente implementación de modelos de esta naturaleza en la administración pública nacional no debería repetir los mismos errores observados en las experiencias internacionales. De este modo, el presente artículo pretende sistematizar el debate actual sobre el tema, a la luz de la literatura sobre las remuneraciones variables, las experiencias internacionales y el conocimiento disponible sobre el contexto brasileño.

La estrategia metodológica ha consistido en la revisión de la literatura centrándose en dos objetivos: a) analizar los estudios que sistematizan los principales argumentos teóricos que explican el fracaso o éxito de la implementación de modelos de incentivos en el sector público -en este punto, hay que señalar los trabajos de Burgess y Ratto (2003) y Weibel et al. (2010) - y b) analizar los resultados de estudios empíricos que hayan investigado casos concretos de implementación de modelos de PRP -a este respecto, hay que señalar los estudios de Marsden (2004), OCDE (2005) y Perry et al. (2009).

Podríamos decir que este trabajo gira en torno a un asunto: ¿Cuáles son las prácticas que llevarán al sistema de remuneración a variable generar buenos resultados dentro de la administración pública brasileña?

\section{LA INEFICACIA DE LOS SISTEMAS DE REMUNERACIÓN VARIABLE EN EL SECTOR PÚBLICO}

La efectividad es el aspecto más debatido en la producción científica mundial sobre los programas de remuneración variable en el sector público. Parece incuestionable que la implementación de un sistema de remuneración variable vinculado al desempeño sea positivo, moderno y eficaz. Sin embargo, estudios realizados en los países de la OCDE apuntan que la remuneración variable en el sector público fracasa o presenta un éxito muy limitado (OCDE, 2005a; Perry et al., 2009; Bowman, 2010; Weibel et al., 2010).

organización de las 60 que implantaron el modelo en todo el Estado. Para más detalles, ver Albergaria, (2010); 
La OCDE (2005b) estudió los modelos exitosos y fracasados de remuneración variable en el área pública de 14 países desarrollados. La conclusión final fue que el PRP motiva sólo a una pequeña parte de los funcionarios, mientras que la gran parte no lo considera un incentivo para el mejor desempeńo. Extensos estudios empíricos realizados en el Reino Unido y en los Estados Unidos mostraron que, a pesar del apoyo a la idea de vincular la remuneración al desempeńo, sólo un pequeño porcentual de los empleados considera que ésta los incita a producir más de lo requerido en el trabajo. En muchos casos, ocurrió un desánimo a la cooperación. Muchos funcionarios públicos, en concreto aquéllos en funciones no gerenciales, consideran el valor del sueldo base y su comparación con los valores de mercado mucho más importantes que los aumentos salariales vinculados al desempeño. La naturaleza y el contenido del trabajo y las perspectivas de desarrollo dentro de la carrera son considerados como los verdaderos incentivos para estos funcionarios. La remuneración variable por lo general no motiva a la mayoría de los funcionarios, independientemente de su configuración (OCDE, 2005b).

Perry, Engbergs y Jun (2009) analizaron las conclusiones de 57 estudios publicados entre los años 1997 y 2008 que trataban de la implementación de sistemas de remuneración variable en el sector público en diversos países. De los resultados, se puede señalar que "la remuneración variable por desempeño en el sector público fracasa por su promesa”, puesto que no se presenta como un incentivo para la mayoría de los colaboradores (a/b) et al., 2009: 43). Para la percepción de los funcionarios, el PRP no es una motivación extra. Para completar, los autores analizaron, de dichos estudios, los 14 trabajos considerados de mayor calidad y encontraron resultados contradictorios. Cuatro de ellos son claramente negativos, cinco no permiten sacar ninguna conclusión y los otros cinco son favorables a la remuneración variable. Estudios más recientes son incluso más pesimistas. Bowman (2010) revisa los resultados de más de veinte años de práctica de remuneración variable en el gobierno federal americano. El autor argumenta que el desempeño de programas de remuneración variable es, como mínimo, decepcionante en relación a las expectativas. El resultado de la implementación resulta a menudo contraproducente. Dos libros publicados recientemente sobre la reforma del servicio civil -Bilmes y Gould (2009) y Donahue (2008) - rechazan la idea de la remuneración variable en los gobiernos. Según Bowman (2010), numerosos estudios insinúan que los individuos no quieren creer que trabajan sólo por dinero. Esto se da especialmente entre los funcionarios públicos. Incluso 
se sienten ofendidos cuando se les trata como si pudieran ser manipulados por los incentivos monetarios. Un argumento semejante al de Bowman (2010) es respaldado por Weibel, Rost y Osterloh (2010). Para ellos, la remuneración variable, en general, acaba con la motivación intrínseca, ${ }^{5}$ provocando una caída del desempeño. Weibel et al. (2010) proponen la suspensión de la implementación de la remuneración variable en el sector público.

Importantes estudios demuestran que las limitaciones de los modelos de remuneración variable en el sector público son serias, repetidas y están relacionadas con las condiciones institucionales, estructurales, ambientales y de implementación.

Entonces, ¿por qué el PRP fracasa en la mayor parte de los casos? Y, ¿por qué, a veces, tiene efectos positivos? Algunas de estas preguntas serán estudiadas a continuación.

\section{El PUNTO DE VISTA DE LOS ECONOMisTaS: LA REMUNERACión VARIAble DESDE LA PERSPECTIVA DE LA ELECCIÓN RACIONAL Y DE LA RELACIÓN AGENTE/PRINCIPAL}

Estudios publicados, especialmente en el campo de la economía, argumentan que los problemas de ineficacia de la remuneración variable en el sector público están relacionados con esquemas de incentivos modelados y administrados inapropiadamente. Weibel et al. (2010) afirman que estos estudios se basan en la teoría de la elección racional, del individuo autointeresado, egoísta y extrínsecamente motivado ${ }^{6}$. Según autores de esta corriente, en situaciones adecuadas el PRP generaría realmente, un aumento en el desempeño de las personas. Sin embargo, existirían problemas típicos de la relación agente/ principal y características estructurales de la administración pública que conducirían a incentivos subóptimos. El objetivo de los investigadores es, por lo

5 Las actividades intrínsecamente motivadas son realizadas porque el individuo las percibe como placenteras, desafiadoras, interesantes y cargadas de propósito. Es decir, en este tipo de motivación, no son necesarios los beneficios externos: el individuo realiza la tarea o actividad porque quiere.

6 La motivación extrínseca es la que se basa en recompensas externas. Se realizan las tareas porque generan compensaciones (como dinero) que pueden, posteriormente, convertirse en productos o servicios que van a cubrir necesidades y, por ello, generar satisfacción. 
tanto, comprender estas situaciones, estudiar las formas adecuadas de modelado e implementación y posibles soluciones para estos problemas.

Este punto de vista considera el PRP desde la relación agente x principal. En esta relación, un actor (principal) delega a otro actor (agente) la realización de una determinada actividad. La relación se dificulta en la medida que el principal y el agente no comparten los mismos objetivos, lo que facilita a estos últimos, actuar de manera contraria a los intereses deseados. Se puede considerar a los funcionarios públicos como agentes de ciudadanos, políticos y liderazgos burocráticos (principales). El principal, en el ejercicio de sus atribuciones y en la búsqueda de su interés, puede exigir una mayor productividad de los funcionarios. Éstos, no tienen que compartir esta aspiración y pueden negarse al desempeño deseado. El problema se agrava cuando el comportamiento o el desempeño del agente es difícil de medirse y el principal cuenta con una información incompleta, insuficiente, para evaluar cómo el agente está actuando en el ejercicio de sus funciones (a/b 1989).

\subsection{LAS CONSECUENCIAS DE LAS MÚLTIPLES TAREAS EN EL SISTEMA DE INCENTIVOS}

Según Burgess y Ratto (2003), las características típicas del sector público dificultan la implementación de sistemas de incentivos. La existencia de múltiples tareas desempeñadas por los agentes, complican por sí solo la estructura de incentivos. Si las acciones fueran sustituibles entre sí (en el sentido de que más tiempo en una actividad significaría menos tiempo en otra), el uso de fuertes sistemas de incentivos puede generar efectos no deseados en los resultados globales. De acuerdo con Segundo MacDonald y Marx (2001), ante la existencia de actividades sustitutas, los agentes preferirán las tareas más fáciles, mientras que el principal querrá que éstos realicen todas las actividades. Los autores afirman que, si el principal no conoce las preferencias de sus agentes, establecer recompensas por desempeño para tareas individuales puede ser subóptimo, ya que puede inducir a que los empleados se dediquen sólo a las tareas más fáciles.

Una posible solución sería hacer que todas las actividades importantes fueran mensuradas por separado, con metas específicas. Así, se le atribuiría una recompensa o una parte distinta de ésta a cada meta. Cuanto más metas cumplidas, mayor la premiación recibida. De nuevo existe el riesgo de múltiples tareas. El peso de la recompensa para cada tarea debe, por eso, calcularse cuidadosamente 
en función de la dificultad para su ejecución y de su relativa importancia dentro de las prioridades de la organización.

\subsection{EL PROBLEMA DE LOS MÚLTIPLES PRINCIPALES}

Una de las características clave del sector público es que los agentes (funcionarios públicos) trabajan para diversos principales (ciudadanos, liderazgos políticos, altos liderazgos de la burocracia, gerencias intermediarias de la burocracia). En estas circunstancias, establecer incentivos adecuados es más complicado, ya que estos principales están interesados sólo en algunos resultados y, también, a veces los intereses de éstos no están alineados. El argumento elaborado por Bernheim y Whinston (1986) es que, en estas circunstancias, cada principal va a ofrecer incentivos positivos a los elementos en los que está interesado e incentivos negativos al resto, mientras que los agentes van a dar preferencia a aquéllos que ofrecen incentivos mayores, dejando de lado a los otros principales y tareas. Una posible solución es intentar negociar con los diversos principales la formalización de los objetivos y metas, dejándoselo claro a los agentes.

\subsection{DifiCULTADES PARA MEDIR LOS RESULTADOS EN EL SECTOR PÚBLICO}

Según Burgess y Ratto (2003), algunos tipos de tareas en el sector público son difíciles de medirlas y recompensarlas. Esto se debe a dos razones: a) en la administración pública, parte de los funcionarios toman decisiones en diferentes actividades, como el caso de los policías, agentes tributarios y asistentes sociales, y b) los funcionarios trabajan para organizaciones que no tienen una meta única, clara y evidente. Para los autores, las dos características juntas hacen más difícil el incentivo y la monitorización de estos individuos.

Teniendo en cuenta esta dificultad de medir los resultados finales, se hace más importante monitorizar el desempeńo durante la ejecución de las actividades, pero de nuevo, las características propias de cada organización pública complican esta tarea. Wilson (1989) estudió diversas organizaciones de gobierno en los Estados Unidos y las clasificó en relación a su posibilidad de medir los medios que los funcionarios usaban y los fines (resultados) que éstas querían alcanzar. En esta clasificación, aparecen organizaciones en las que ni los resultados ni los 
medios son fáciles de medir ${ }^{7}$. En éstas, los burócratas controlan la distribución de los bienes y los servicios para los clientes y sus decisiones dependen de factores variables que no son fácilmente observados por sus superiores. El cuerpo de policías es un ejemplo típico: La decisión de detener a una persona se basa en la sospecha inmediata de culpa, lo que no es observable por sus superiores. Y la decisión de transferir un bien o prestar un servicio no pude basarse en un valor pagado por el cliente, lo que sería fácilmente observable. En estas organizaciones, en las que ni el esfuerzo del agente ni el resultado son fácilmente observables, la medición del desempeño es un desafío y un problema habitual.

Burgess y Ratto (2003) argumentan que, en estas situaciones, las informaciones sobre el desempeño están disponibles solamente de forma conjunta. Los resultados son producidos por individuos, pero se miden teniendo como referencia el grupo de individuos (unidad gerencial): De esta forma, el objetivo en equipos es especialmente relevante como una solución para la medición del desempeño en el sector público.

\subsection{TiPOS DE MEDICIÓN DEL DESEMPEÑO Y DEL MODELADO DE INCENTIVOS}

Gehardt (2009) afirma que, en función del tipo de medición del desempeño, los modelos de remuneración variable se dividen en: a) basados en el comportamiento, que evalúan subjetivamente el cumplimiento de determinados requisitos, tales como la percepción de la dirección en relación con el trabajo en equipo, compromiso, etc., llamados de remuneración por mérito basados en los resultados objetivamente mensurables, llamados de remuneración por desempeño.

Para este autor, el modelo basado en el comportamiento tiene como ventaja potencial el hecho de poder utilizarse en cualquier tipo de situación, además de cumplir requisitos que están dentro de la gobernabilidad del funcionario, siendo competencia de éste tomar las medidas oportunas para mejorar el desempeño. Burgess y Metalcafe (1999) afirman que este tipo de medida es más adecuado cuando los resultados son más difíciles de medirse. Sin embargo, el carácter subjetivo de este tipo de medida hace que sea más difícil justificar las diferencias de desempeño entre los empleados (a/b, 2009). Este aspecto, como se podrá

7 Wilson (1989) las llamó de coping organizations. 
constatar a lo largo de este trabajo, es especialmente preocupante en el caso brasileńo, lo que hace que este tipo de medición sea poco adecuada en Brasil.

Si bien, el modelo basado en los resultados es percibido como más justo entre los empleados, debido a su carácter objetivo. Por este motivo, se utiliza más éste cuando se trata de incentivos relevantes. Burgess y Metalcafe (1999) afirman que es el más adecuado para situaciones en las que los resultados son más fáciles de medirse. También hay algunos aspectos negativos en esta modalidad: No permite una medición adecuada de los resultados de todas las tareas y la falta de medición de algunos aspectos de la tarea puede provocar que los empleados los ignoren. Además, cuando se da un desempeño desfavorable, en el caso que los empleados lo atribuyan a factores externos, los cuales no controlan, puede generar reacciones negativas y presiones para revisar las metas (a/b, 2009). A pesar de estas críticas, el argumento de Barbosa (1996), presentado en este estudio, indica que este modelo es el más adecuado para el caso brasileño, debido a su objetividad.

\subsection{EL TRABAJO EN EQUIPO Y SU RELACIÓN CON LOS INCENTIVOS}

Holmström (1982) argumenta que, en un equipo donde dependen unos de los otros para producir resultados, aparece el problema del "polizón" (free rider). Este problema se agrava cuanto más grande es la organización. En esta situación una buena recompensa depende de la facilidad de medición del resultado y también del tamaño del equipo. Cuanto mayor sea la dificultad a la hora de medir los resultados, más compleja será la elaboración de un buen sistema de incentivos. El problema del free rider es la explicación más común de la Economía para justificar la falta de éxito con los sistemas de recompensa por equipos (Burgess y Ratto, 2003). Dentro de esta perspectiva, en el trabajo en equipo, una situación que puede estimular el desempeño es la monitorización por los iguales (colegas de trabajo). Vincular las recompensas al desempeño del grupo estimula esta monitorización y, por consiguiente, reduce la práctica del free rider. Kendel y Lazear (1992) muestran que una mayor monitorización por los iguales puede inducir a un mayor esfuerzo de los individuos por el riesgo a ser cogidos "haciendo el vago" sea mayor. Esto tiene una implicación en el tamaño de los equipos, pues en los equipos muy grandes es más difícil esta monitorización por parte de los iguales. En el análisis de Holmström 
(1982), el equipo se define como una unidad de producción, en el que sus miembros contribuyen para conseguir un resultado en común.

Hay un importante debate sobre el modelado de los sistemas de incentivos entre los defensores de evaluaciones basadas en los individuos y las basadas en los equipos. Gehardt (2009) señala que, en cuanto al nivel de medición del desempeño, los modelos de remuneración variable se dividen en individualistas, colectivos o híbridos (donde la remuneración se basa en parte en el desempeño individual y en parte en el desempeño colectivo). Aunque la perspectiva económica presenta riesgos con la utilización de modelos colectivos, éstos están concretamente ganando fuerza en las experiencias de PRP en los gobiernos. Los modelos basados en individuos, aunque promuevan un fuerte incentivo debido al vínculo directo del desempeño individual con la premiación, también presentan aspectos negativos, principalmente relacionados con la cooperación. Gehardt (2009) argumenta que el uso de modelos de incentivos individuales puede fomentar la desintegración y entorpecer el trabajo en grupo, en la medida en que se evaluará y recompensará a cada funcionario de forma individual y el resultado colectivo de trabajo puede dejar de ser una prioridad. Los estudios de la OCDE (2005a, 2005b) y de Perry et al. (2009) concluyen que los modelos basados en equipos parecen presentar mejores resultados que los basados en evaluaciones individuales de desempeño.

Una de las principales consecuencias del informe Makinson (2000), que sugirió modelos de incentivos para la administración pública británica, fue la aparición de sistemas de remuneración variable basados en equipos. Estos equipos variaban desde 100 funcionarios hasta miles de empleados de divisiones enteras. Burgess y Ratto (2003) argumentan que una definición clara del equipo es muy importante para la configuración de un sistema de incentivos exitoso. En este sentido, los equipos pueden definirse en función del proceso de producción o, estar "forjados artificialmente", para proporcionar un estímulo a la cooperación entre las distintas unidades. Debe haber una lógica en la definición del equipo y esto debe estudiarse caso por caso. Por ejemplo, si el objetivo es generar incentivos y el control de los iguales (colegas de trabajo), evitando de esta forma el free rider, en la configuración del equipo debe considerarse este aspecto, particularmente en lo referente al tamaño del grupo, debiendo ser relativamente pequeño. 


\section{LAS INTERFERENCIAS DEL ASPECTO PSICOLÓGICO EN LA REMUNERACIÓN VARIABLE}

Perspectivas más cercanas a la psicología consideran que el fracaso de los sistemas de PRP no se debe a los incentivos subóptimos, sino a la motivación de los empleados. Según Weibel et al. (2010), las teorías económicas basadas en el autointerés no cuentan con una estructura analítica suficiente para analizar la motivación de los empleados, especialmente los empleados de la administración pública. El uso de la remuneración variable generaría efectos contrarios en los funcionarios públicos, muchas veces disminuyendo su motivación.

Las modernas teorías psicológicas se basan en la idea de que existen dos componentes en la motivación: La motivación intrínseca y extrínseca. Observar el peso de estos dos componentes en la motivación de los individuos parece un punto importante para poder comprender el impacto de la remuneración variable en el desempeńo de cada profesional. De acuerdo con Weibel et al. (2010), en la administración pública los funcionarios tienen, por lo general, una motivación intrínseca considerable, realizando las tareas en función de su sentido del deber, lealtad y placer, o sea, realizan estas actividades pues creen en ellas y, al hacerlas, tienen la sensación de cumplir con su deber. Esta motivación intrínseca, en ciertas situaciones, puede disminuir al introducir la remuneración variable. Dar a alguien incentivos financieros para realizar una serie de tareas que podrían ser hechas por mero placer desmotiva, en la medida en que la persona pasa a ver la tarea como algo controlado por incentivos externos y no por placer, por propia voluntad. Por lo tanto, estos incentivos financieros pueden provocar costes ocultos y, así, disminuir el desempeño (a/b et al., 2010). A este efecto de la disminución o neutralización de la motivación intrínseca en función de la existencia de recompensas extrínsecas se le conoce en la literatura como efecto desplazamiento o expulsión (crowding-out effect). En otras palabras, el aumento de la motivación extrínseca por medio de mecanismos externos (recompensas) provoca el "desplazamiento" o la "expulsión" de la motivación intrínseca. Se le atribuye a este efecto buena parte de las limitaciones motivacionales de la remuneración variable. 


\section{1. EL EFECTO CROWDING-OUT ${ }^{8}$}

Varios autores han intentado comprender el problema del efecto crowdingout. Osterloh y Frey (2002) sintetizan argumentos teóricos y resultados de las investigaciones empíricas sobre el asunto. Existen dos puntos de vista sobre este efecto.

Según el punto de vista de la teoría de la evaluación cognitiva, dicho efecto hace que la motivación intrínseca sea substituida por la intervención externa, lo que se percibe como una restricción de la autonomía del individuo. El individuo en cuestión no se siente más responsable por una determinada tarea, ya que la tarea es "controlada" e incentivada por dinero, por la premiación variable. La tarea pasa a ser controlada por el dinero más que por el placer. El individuo pasa, por lo tanto, a atribuirle la responsabilidad de la tarea a quien está pagándola, librándose de gastar energía en su realización.

Ahora, la teoría de los contratos psicológicos argumenta que cada relación de trabajo incluye un aspecto extrínsecamente motivado (dinero) y un aspecto relacional entre las dos partes. En el caso de que la parte relacional del contrato se rompa, la buena fe recíproca se refleja en el pago. Evidencias empíricas demuestran que, cuando esto sucede, las partes del contrato perciben que la realización de la tarea se convierte en una simple relación comercial. Por ejemplo, cuando un supervisor felicita a un empleado por un gran esfuerzo con un regalo simbólico (flores por ejemplo), la motivación intrínseca de este empleado tiende a aumentar por que éste siente que su esfuerzo es valorado. Sin embargo, si por alguna razón, este empleado percibe que el gesto del superior es un objetivo instrumental, su motivación intrínseca se ve afectada negativamente. Las flores pasan a ser percibidas como controladoras y la relación es interpretada como algo comercial, disminuyendo la motivación (a/b, 2002).

Se han realizado varios análisis empíricos en relación con el crowding-out effect. Uno de los más relevantes (Eisenberger y Cameron, 1996) analizó un total de 59 artículos producidos entre 1971 y 1997 y concluyó que las recompensas disminuyen la motivación intrínseca en las actividades consideradas interesantes (aquellas que los experimentos mostraron como intrínsecamente controladas) de manera altamente significante y confiable. Osterloh y Frey (2002) argumentan,

8 La traducción al español sería "efecto de desplazamiento, expulsión o exclusión”. 
por lo tanto, que no hay duda de que el crowding-out effect existe y es un fenómeno significativo bajo ciertas circunstancias. Por lo tanto, hay que calcular el resultado neto entre efecto y premiación (derivado de la recompensa) y restarle el efecto crowding-out, para evaluar de hecho el impacto de la remuneración variable sobre la motivación.

\section{2. LA COMPARACIÓN ENTRE LOS ESTÍMULOS INTRÍNSECOS Y LOS EXTRÍNSECOS}

La perspectiva de la psicología expone que la remuneración variable disminuye el desempeño en el caso de las tareas interesantes. Por lo contrario, la perspectiva de la economía argumenta que la remuneración variable aumenta el desempeńo independientemente del tipo de tarea, si el sistema de incentivos está modelado e implantado correctamente.

Nuevos estudios publicados por Weibel et al. (2010) alumbran está supuesta contradicción. Estos autores revisaron las conclusiones de 46 estudios publicados en diferentes revistas académicas de Economía y Psicología. Los estudios se clasificaron en función de su referencia a actividades interesantes o no interesantes. Los autores concluyeron que la remuneración variable provoca un aumento del desempeño significativamente en el caso de las actividades no interesantes, mientras que en el caso de las actividades interesantes el desempeño disminuye. En el estudio, no se encontraron diferencias significativas entre los estudios publicados en las revistas de economía y psicología. De esta forma, podemos articular las dos perspectivas (económica y psicológica) para llegar a una única conclusión. La Económica argumenta que gran parte de los problemas de la remuneración variable está relacionada con los incentivos modelados inadecuadamente. La Psicológica completa está explicación añadiendo un aspecto: PRP funciona bien con las actividades no interesantes y presenta limitaciones serías con las actividades interesantes, pudiendo, incluso, provocar una disminución en el desempeño del funcionario.

Estas conclusiones producen importantes consecuencias en el modelado de los incentivos en el sector público. El estudio de la OCDE (2005a) demostró que los incentivos variables tienden a ser, como máximo, un $10 \%$ de la remuneración para los niveles no gerenciales. Debido a esto, el efecto premiación es pequeño 
en la administración pública ${ }^{9}$. Sin embargo, el efecto crowding-out es alto, ya que el contenido del trabajo y la motivación intrínseca parecen ser importantes elementos de incentivo en la administración pública, hecho que es confirmado por varios autores ${ }^{10}$. Un bajo efecto premiación sumado a un alto efecto crowdingout produce una caída del desempeño, especialmente en las tareas interesantes.

Para Weibel et al. (2010), esta realidad sintetiza la mayor parte de los motivos del fracaso de la remuneración variable en el sector público. En este ambiente, los funcionarios de niveles operacionales son más propensos a considerar sus trabajos menos interesantes que los gerentes ${ }^{11}$ y por ello, son buenos candidatos para la remuneración variable. Ahora, para los gerentes, el PRP sería una estrategia arriesgada y potencialmente negativa, ya que la función gerencial tiende a ser interesante debido al desafío y la complejidad de la tarea. Estas afirmaciones teóricas parecen concordar con los estudios empíricos. Perry et al. (2009) afirman, después de analizar la efectividad de las experiencias de PRP, que éstas parecen presentar más éxito en los niveles operacionales, donde las tareas son menos ambiguas y los resultados más concretos y medibles, lo que contradice la suposición de que serían más efectivas en los niveles gerenciales.

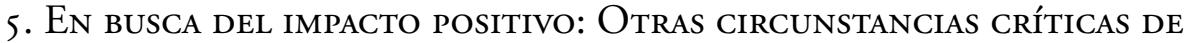 LA IMPLEMENTACIÓN}

La mayoría de los estudios resalta, no obstante, que la remuneración variable en el sector público puede presentar efectos positivos (OCDE, 2005; Perry et al., 2009; Marsden, 2010; Burgess y Ratto, 2003). El modelo no es un mero fracaso. Sus limitaciones, aunque graves y repetitivas, pueden ser abordadas o minimizadas para obtener resultados positivos.

Según Marsden (2010), aunque la remuneración variable pueda tener efectos negativos sobre la motivación bajo algunas circunstancias, existen aportaciones

9 Una serie de autores confirma esta afirmación: Ingraham, (1993). Kellough, and Haoran Lu (1993). y Moon, M Jae (2000).

10 Para más información sobre este argumento, véase: François, (2000). Frank, Sue A., and Gregory Lewis. 2004. y Grand, (2006).

11 Buelens, Marc y Van Der Broeck, Herman (2007). 
para mejorar el desempeño de las organizaciones. La medición del desempeño y la definición de metas (que generalmente son fortalecidas con la implementación del PRP) han contribuido a la mejora del desempeńo. Este autor afirma que la implementación de la remuneración variable ha incentivado la renegociación de prioridades y modelos de desempeño entre los empleados y la dirección. Una vez que las metas y objetivos de las organizaciones son la base para calcular la parte variable, hace falta que éstos sean aclarados, comunicados, negociados y repactados con los empleados. La relación contractual entre el jefe y el subordinado se rediscute periódicamente, lo que favorece a la convergencia de las metas de ambas partes (individuo y organización).

Los estudios refuerzan una importante constatación: Discutir la relación entre remuneración variable y la mejora del desempeño no implica, sólo, comprobar el posible aumento del esfuerzo de los empleados. Hay que comprobar, también, el grado de esfuerzo redirigido a los objetivos deseados (Gerhart, 2009). La simple redirección del esfuerzo (incluso sin que éste aumente) a favor de las prioridades de la organización puede generar una mejora en el desempeño. Incluso cuando el PRP no genera más esfuerzo, puede provocar un esfuerzo de mayor calidad, o sea, puede mejorar la alineación entre el individuo y la organización. Estas afirmaciones guardan relación con importantes estudios recientemente publicados. Los trabajos de Perry et al. (2009) y OCDE (2005a, 2005b) concluyeron que el PRP puede mejorar el desempeño, por lo general, sin ser a través de la motivación. Los buenos resultados son debidos a los efectos derivados como la alineación de esfuerzos en torno a las prioridades, la medición y la evaluación del desempeño, mejor toma de decisiones y mayor cooperación cuando las metas son colectivas.

La literatura señala, sin embargo, que estos efectos positivos suceden cuando se dan circunstancias apropiadas para la implementación. Pero estas circunstancias parecen estar presentes en pocas de las experiencias investigadas. Como la gran parte de los autores se muestra reticente y pesimista en lo que se refiere a los resultados de la remuneración variable en la administración pública, es necesario perfeccionar los modelos y procesos de implementación de la remuneración variable para fortalecer sus aspectos positivos. A continuación, se discutirán otros aspectos relevantes del modelado y la implementación. 


\subsection{Primeros Pasos PARA La INTROdUCCión DE Sistemas de PRP}

Kerr (2004) afirmó que para que la gestión sea efectiva, el establecimiento de las recompensas debe relegarse a un tercer lugar. En un segundo lugar debe medirse el desempeńo y, tanto las recompensas como la medición deben subordinarse a una definición clara y concreta de lo que debe ser hecho. El primer paso, por lo tanto, deber ser la elaboración de un plan estratégico para la organización. Este puede definirse sobre el papel con las diez prioridades para el año o sobre un mapa estratégico elaborado con el apoyo de una buena consultoría. Además, según el autor, lo más interesante de este principio es que éste, por muy evidente que sea, es vulnerado sistemáticamente. Kerr (2004) muestra que el establecer indicadores de desempeño y metas para comprobar el cumplimiento (o no) de las prioridades definidas por la organización debe hacerse antes de implantar un sistema de PRP. La ausencia de un plan estratégico con metas para la organización anteriores a la introducción de los sistemas de incentivos puede provocar el establecimiento de metas sin lógica, sólo para poder implantar el PRP, desvinculadas de un proceso mayor, estipuladas sólo para sectores (o carreras) específicos de la organizaciones y dirigidas a objetivos no muy nítidos (ya que no se discutieron las prioridades y las estrategias para la organización en conjunto). De esta forma aparecen situaciones como la descrita por Kerr (1975), o sea, la organización puede recompensar cosas equivocadamente e, incluso, aquello que quiere evitar.

\subsection{LA DEFINICIÓN DE LOS INDICADORES DE DESEMPEÑO}

Según Pacheco (2009), varios autores han discutido el asunto de la medición de los productos/servicios o impactos. Se entiende por productos/servicios a aquéllos que son ofrecidos o prestados en nombre del gobierno por los ministerios/ secretarias a individuos u organizaciones externas (Trosa, 2001). Se les conoce en la literatura como outputs. Los productos/servicios están directamente vinculados con las políticas públicas, como por ejemplo, el número de atenciones preventivas en los ambulatorios. Ahora, los impactos -también llamados de outcomes- son los cambios efectivos deseados por la sociedad como resultado de las políticas públicas, como por ejemplo la reducción de la mortalidad infantil de una determinada población. 
Los productos/servicios son muy importantes para evaluar el resultado de la política pública de forma más específica y concreta, estableciendo una relación entre los procesos y los resultados. Existen los argumentos tradicionales: Los defensores de los productos/servicios argumentan que éstos son más fáciles de medirse, que son una consecuencia directa de las políticas públicas y, por lo tanto, miden mejor el desempeńo. Los defensores de los impactos argumentan que son éstos los que realmente miden si la política está funcionando, aunque sólo sean detectados a medio/largo plazo.

Trosa (2001) presenta una solución pragmática: productos/servicios e impactos son ambos importantes y responden a diferentes preguntas. Para la autora, la medición de los productos/servicios permite conocer lo que se produce realmente con los recursos públicos y los impactos permiten indagar sobre la eficacia y la utilidad de lo producido. Los gobiernos deben empezar por los productos/ servicios cuya medición es más fácil, y avanzar en dirección a los impactos, a través de la construcción de una cadena lógica que conecte las acciones a los objetivos deseados, relacionando impactos, resultados intermediarios y acciones.

Para Behn (2003, 2004a), no basta con discutir la medición de productos/ servicios o impactos. Hay que explicar cuáles son los propósitos de la medición, pues cada objetivo requiere una medida diferente. Según el autor, para motivar a las personas, la organización necesita medidas en tiempo real, pues la recompensa sólo tiene sentido si se monitoriza los resultados a tiempo para poder corregirlos. Behn defiende fervientemente la medición de productos/servicios, especialmente cuando el objetivo es la motivación. Por lo tanto, cuando se habla de remuneración variable por desempeño, la literatura nos aclara que centrarse en la medida de productos/servicios es más eficaz, pues crea las circunstancias propicias para corregir directrices y también genera la percepción, por parte de los funcionarios, de que sólo con cambiar el comportamiento se mejoran los resultados, ya que estos resultados son una consecuencia directa de la acción de la organización.

Según Pacheco $\left(2010^{12}\right)$, en Brasil parece haber una tendencia a sobrevalorar los impactos. Cada vez se habla más de impactos, como si éstos representasen la cara más moderna de la gestión por resultados en la administración pública. Sin

12 Regina Pacheco, conferencia de clausura, congreso CONSAD 2010, Brasília-DF. 
embargo, aunque sean medidas importantes y éstas deban medirse, restringirse a los impactos provoca graves limitaciones. Éstos pueden tardar mucho tiempo (a veces años) para manifestar el resultado de las acciones. Además hay diversos factores que influyen en los impactos. Por ejemplo, una mejora en las condiciones de salud de la población no depende solamente del número de atenciones preventivas. Pues también influyen el modelo de saneamiento, el nivel de educación e higiene y otros factores. Aunque la medición de estos impactos sea importante y necesaria para una serie de objetivos, la utilización de éstos en los sistemas de PRP debe ser limitada.

\subsection{El PAPEL DE LA DIFICULTAD Y EL VOLUMEN DE LAS METAS EN EL DESEMPEÑO}

Locke y Latham (2002) afirman que las metas difíciles producen mejores resultados. Se constató una correlación lineal positiva y altamente significativa, donde las metas más difíciles (en la percepción de quien debe cumplirlas) producen mayores niveles de esfuerzo y desempeño. De acuerdo con los autores, esta relación entre metas difíciles y desempeño ha sido confirmada con 35 años de estudios y comprobada con más de 100 tareas diferentes involucrando a 40 mil participantes en 8 países, tanto en el laboratorio como en los estudios de campo. Estos resultados pueden aplicarse a individuos y grupos (A/B, 2002). Los autores también compararon los efectos de la definición de metas difíciles y específicas con la simple utilización del incentivo moral "da lo mejor de ti". Los resultados demuestran que las metas difíciles, específicas y claramente definidas producen un desempeño superior que un simple incentivo moral verbal.

Otro punto importante sería que las metas en exceso producen una "sobrecarga cognitiva”. A menos que la mayor parte de éstas pueda ser delegada, un único gerente, tiene condiciones para administrar entre tres y siete metas, dependiendo de la complejidad o del tiempo que éstas demandan para lograrlas (LOCKE, 2004). Los autores también argumentan que, para que las metas sean efectivas, las personas necesitan feedbacks periódicos que muestren la evolución del desempeńo. Si éstas no saben cómo están desempeñando su función, es prácticamente imposible ajustar o redireccionar el esfuerzo y las estrategias para logar las metas. Éste es un argumento más a favor de los productos/servicios, en detrimento de los impactos para apoyar los sistemas de incentivos. 


\subsection{LOS RIESGOS ASOCIADOS AL FENÓMENO GAMING EN LOS SISTEMAS DE PRP}

La palabra gaming se refiere a aquellas situaciones donde los subordinados se aprenden "las reglas de juego" y empiezan a "jugar" para conseguir sus intereses, incluso contrariando los objetivos del sistema. Se trata de una manipulación y mal uso del sistema, particularmente por parte de los subordinados (agentes). Cualquier modelo de incentivos que se base en la evaluación de indicadores y metas está sujeto a este fenómeno, lo que representa una limitación y un riesgo para los sistemas de remuneración variable.

La literatura está llena de ejemplos de este fenómeno. Hood (2006) señala que, en Inglaterra, se dieron situaciones en las que se solicitó a los pacientes que aguardaran dentro de las ambulancias (evitando que pasaran por recepción) hasta que el hospital tuviera garantías de poder atenderlos dentro de la meta de 4 horas de espera. También hubo casos donde los profesores entrenaban a conciencia a sus alumnos para los exámenes que evaluaban el desempeño de la escuela, dejando de lado cualquier otro contenido o materia que no entrase en este examen; incluso, hay relatos de alumnos flojos "apartados", con la perspectiva de centrarse en aquéllos que van a hacer mejorar el desempeño de la escuela en el examen (Van Dooren, 2008).

Los incentivos malintencionados aparecen generalmente cuando el trabajo exige una serie de tareas, pero solamente algunas son medidas y recompensadas. En este caso, el trabajador concentra sus esfuerzos en las recompensadas, en detrimento de los objetivos de la organización. Robert Behn (2007) alerta a los gestores públicos sobre este punto al afirmar que pocas medidas concentran la atención de las personas, lo que significa una clara ventaja y, al mismo tiempo, un inconveniente. Para este autor, lo que no se mide no se hace y, por lo tanto, los gestores deben analizar cuidadosamente lo que debe establecerse como medida y, concretamente, qué medidas deben ser la base para los sistemas de incentivos.

Según Hood (2006), los especialistas identificaron como mínimo tres tipos de gaming y comportamientos oportunistas en torno de las metas. El más conocido se da cuando los subordinados (agentes) perciben que las metas futuras se verán influidas por el mayor desempeño logrado en el pasado (rachet effect o efecto de bola de nieve). De este modo, empiezan a pensar que si trabajan mucho durante un año se perjudicarán ellos mismos puesto que la meta del próximo año será 
mayor. Por lo tanto, los agentes tienden a disminuir la productividad en el período actual para sentirse menos presionados en el futuro. El segundo tipo es conocido como el efecto umbral (threshold effect). Éste se da cuando se imponen metas iguales a todas las unidades del sistema, haciendo que no haya incentivos para destacar e, incluso, hacen que aquéllos que tienen mejor desempeño disminuyan la cantidad y calidad de sus servicios hasta los niveles más bajos definidos por la meta. El tercer tipo de gaming consiste en la distorsión de los objetivos — "hitting the target and missing the point": "lograr las metas sin sentido"- como, por ejemplo, situaciones en las que el tiempo de respuesta se reduce con la consecuente pérdida de calidad en la atención. En estas circunstancias, el proceso de definición de metas se asemeja a un juego entre el agente y el principal, donde gana quien sea más astuto. En este juego se pierde el objetivo del mejor desempeño.

En la elaboración e implementación de un sistema de remuneración variable es más importante evitar crear incentivos que puedan provocar esta situación que intentar reducir el gaming después de constatar su presencia. La aparición del gaming se relaciona con dos aspectos: La presión ejercida en los individuos debido al sistema de metas, por un lado, y la presencia de mecanismos anti-gaming, por otro.

La existencia de una remuneración variable vinculada a la evaluación de los resultados o a un sistema de monitorización de metas provoca más presión e incentiva que las personas practiquen el gaming. Una situación muy frecuente es cuando hay una gran exigencia de metas, como en el caso inglés de la administración de Tony Blair (en la que los gestores sufrían una fuerte exigencia por parte del ejecutivo ${ }^{13} \mathrm{y}$ hasta se les despedía si los resultados eran bajos). En este sentido, el valor o peso monetario de la remuneración variable supone un cambio. De acuerdo con la OCDE (2005b), el valor monetario del PRP en el sector público en los países desarrollados se ha establecido como máximo en un $10 \%$ del sueldo, para los funcionarios, y un $20 \%$ para los niveles gerenciales. El hecho de no sobrepasar este límite parece ser importante para evitar el "todo vale" para conseguir los resultados, lo que incentivaría la práctica del gaming.

Los mecanismos anti-gaming ayudan a disminuir la presencia de este fenómeno. Hood (2006) cita la definición de especificaciones detalladas en relación con

13 Para más información sobre el funcionamiento del sistema inglés véase, Steven (2006). 
los indicadores y metas, con la realización de auditorías y el castigo ejemplar de subordinados malintencionados. Para Locke (2004), una buena forma de prevenir este tipo de comportamiento es hacer que las reglas de conducta, normas éticas y modelos de comportamiento estén claramente notificados y reforzados por la dirección. Existen otras medidas para disminuir el efecto bola de nieve. El principal puede buscar medios para mejorar el conocimiento sobre la ejecución de la tarea, disminuyendo las desigualdades en la información y profundizando en el conocimiento sobre el nivel de esfuerzo empleado en la producción. Además, el principal puede comparar el desempeño de su unidad de producción con la de otras similares en la misma organización u otras organizaciones, el llamado benchmarking.

La definición de metas puede, por sí misma, disminuir el gaming. El pacto de resultados puede hacerse por "conjunto de indicadores", en aras de completar, en estos indicadores, cantidad y calidad, generando incentivos para evitar efectos malintencionados. Las metas de reducción del tiempo de espera de los servicios, por ejemplo, deben estar acompañadas de indicadores de calidad y satisfacción de estos servicios.

Sin embargo, la aplicación de estos mecanismos anti-gaming demanda un análisis, estudio, diálogo y conocimiento de las políticas públicas. La existencia de una unidad de inteligencia compuesta por funcionarios capacitados, vinculada directamente al principal y que lo ayude en los detalles de la negociación con el agente parece ser algo fundamental. En la relación agente x principal, solucionar los problemas de desigualdad en la información puede ser hasta imposible pero, implantar mecanismos para reducirlos parece ser algo necesario.

\subsection{LA CULTURA BRASILEÑA Y SU INFLUENCIA SOBRE LOS RESULTADOS DEL SISTEMA DE PRP}

Barbosa (1996) demostró que hubo varios intentos de implantar sistemas de evaluación individual por méritos en Brasil a lo largo del tiempo y las presiones sociales hicieron que estos modelos fracasaran siempre. Aunque todos apoyen esta lógica meritocrática, la práctica social no la legitima. La autora argumenta que el concepto de igualdad en Brasil es diferente al existente en los EE.UU. Donde la idea de igualdad significa un tratamiento igual ante la ley, no supone necesariamente la búsqueda de un estado sustancialmente igualitario. La diferencia entre los individuos está reconocida, se legitima y se percibe como el resultado de un talento 
diferente - aptitud innata - , que permite a algunos individuos realizar y practicar ciertas acciones con mejor desempeño que otros, en función de su capacidad y deseo en realizarlas. Se perciben las diferencias como algo inevitable y deseado. La sociedad americana está preparada para admitir la igualdad jurídica y la desigualdad de hecho, en función de las diferencias por desempeño individual. En este contexto, el desempeńo funciona como un mecanismo socialmente legítimo, que le permite a la sociedad distinguir, evaluar, jerarquizar y premiar a los individuos.

El concepto de igualdad en Brasil es diferente. Barbosa (1996) afirma que el desempeño se entiende más como un resultado del ambiente que como un esfuerzo del individuo. Debido a esto las producciones individuales no se comparan, pues se ve al producto de cada una como el fruto de las circunstancias históricas subjetivas. Para la autora, los brasileños atribuyen un desempeño bajo a la falta de oportunidades en la vida, no legitiman el mérito como un producto natural de la diferencia social; en resumen: "En Brasil, el desempeño no se evalúa, se justifica". Por esto el síndrome de la isonomía, los ascensos automáticos para todos y la inflexibilidad de la administración pública en la que las diferentes categorías funcionales están amarradas unas a las otras, de forma que cualquier intento de distinción, incluso basado en la diferencia de funciones, se ve como una concesión de derechos que debe generalizarse, lo que produce el famoso efecto cascada. En la sociedad brasileña el establecimiento de grados o jerarquías se percibe como la introducción de desigualdades, lo que está en contra de los propósitos del sistema. En este contexto, la lucha por el reconocimiento del mérito individual es algo extremadamente difícil y polémico. Especialmente cuando la evaluación conlleva impactos financieros, el problema se agrava. Incluso cuando hay evaluaciones objetivas sobre los distintos desempeños, en el momento de la distribución financiera, ésta acaba siendo igualitaria. Esto se justifica normalmente con la poca cantidad de dinero disponible y así es mejor "dar un poco para cada uno para que todos reciban algo". Así que todos son evaluados positivamente o no se evalúa a nadie. (a/b, 1996).

Las consecuencias de este análisis son impactantes para el modelo y para el posible éxito de los sistemas de PRP en Brasil. Aunque el argumento de Barbosa esté dirigido a la evaluación del desempeño individual, cualquier modelo de incentivos se basa en esta parte individual o la evaluación colectiva para medir la remuneración variable. El síndrome de la isonomía y la búsqueda de una 
igualdad hacen que, incluso existiendo distintos desempeños, haya una presión social que provoque que todos sean recompensados de forma igual. Esto produce otras presiones en el sistema de evaluación porque la cultura brasileña atribuye la desigualdad en el desempeño a la falta de oportunidades en la vida. De este modo, no tiene sentido dar una nota que no sea un 10 , pues cada uno hace lo que puede. La implementación de los sistemas de PRP en Brasil debe tener en cuenta este aspecto cultural y, así, prever los mecanismos que hagan lo más objetivo posible esta diferencia en los desempeños. Por este motivo los modelos que se basan en resultados medidos de forma objetiva son más adecuados al caso brasileño que los modelos estructurados en evaluaciones subjetivas del desempeño.

\subsection{LOS EFECTOS DE LA CREDIBILIDAD Y DEL SENTIDO DE JUSTICIA}

La imagen que los empleados tienen sobre el funcionamiento del sistema de remuneración variable influye en el fracaso o éxito del modelo. Perry et al. (2009) concluyeron que el éxito del PRP depende de la percepción que tienen los empleados del sistema, si es justo, transparente y no político. Se consideraron factores críticos para el éxito del modelo, el nivel de confianza y la consistencia del sistema de evaluación. Si los empleados perciben que las reglas del sistema se han relativizado, flexibilizado o manipulado en beneficio de alguien, el sistema perderá su credibilidad. Los autores también argumentan que la credibilidad en el liderazgo parece tener un papel relevante para transmitirle al empleado sentido de justicia en relación al PRP. Gehardt (2009) y Dahlström y Lapuente (2010) corroboran también la importancia de que los empleados confíen y crean en las reglas para que el modelo sea efectivo.

\section{LA ELECCIÓN DE MODELOS APROPIADOS PARA LOS SISTEMAS DE INCENTIVOS EN EL CASO BRASILEÑO}

El estudio de las experiencias en las distintas publicaciones mencionadas en este trabajo y los argumentos teóricos propuestos por los autores señalan las posibles prácticas que conducirían al sistema de remuneración variable a generar buenos resultados en la administración pública brasileña. A continuación, se describe una 
propuesta preliminar de estas prácticas, que pueden y deben ser reforzadas por la literatura gerencial brasileña.

Como se observa en la literatura, para que el PRP realmente contribuya a la mejora del desempeño, hace falta garantizar que el modelo incentive el comportamiento y los resultados en la dirección correcta. Las metas creadas sólo para realizar los cálculos de la remuneración variable no se toman en serio. Dentro de las experiencias con éxito la remuneración variable es una parte de un sistema más amplio de gestión de resultados y metas, donde las informaciones del desempeño son monitorizadas y utilizadas para tomar decisiones diariamente. En estas circunstancias el potencial del sistema se utiliza plenamente, puesto que se hace un seguimiento de los incentivos y se redireccionan a medida que las prioridades específicas de la organización se van identificando. De esta forma se espera que la práctica 1, presentada a continuación, genere buenos resultados en el contexto brasileño:

\section{Práctica 1: La primera etapa indispensable para la elaboración de un sistema eficaz es la construcción de una agenda estratégica (con indicadores y metas) para toda la organización (y no sólo para algunas partes de ésta)}

Según señala la literatura, es arriesgado estructurar el modelo de PRP en torno a la evaluación de comportamientos pues presenta un alto grado de subjetividad. El motivo principal es el riesgo de benevolencia. De este modo, es más aconsejable estructurar la medición en torno a los productos/servicios. Estos son más apropiados para redireccionar los esfuerzos en busca de resultados, pues pueden medirse prácticamente en tiempo real. También son más apropiados para representar, de hecho, los esfuerzos emprendidos para la ejecución de las políticas públicas. Los productos/servicios "ven" mejor y directamente el desempeño, lo que les hace más útiles para la toma de decisiones y la gestión diaria. Diversos estudios señalan que las personas necesitan sentir que son responsables por las metas para sentirse motivados. Una pequeña parte de la composición variable puede vincularse a los impactos, sólo para que éstos ejerzan la función de instigadores y para que el ejercicio de repensar la relación procesos $\mathrm{x}$ resultados se mantenga constante. De esta forma se espera que la práctica 2, presentada a continuación, genere buenos resultados en el contexto brasileño: 
Práctica 2: El modelo debe basarse únicamente en la evaluación de resultados objetivamente cuantificables. Los resultados deben privilegiar más a los productos/servicios y menos a los impactos.

Los sistemas basados en equipos han destacado como más eficaces dentro del sector público, tanto por la dificultad de medir los resultados a nivel individual, como por su refuerzo a la cooperación. No hay una única fórmula para la definición de un equipo. Hay que evaluar caso por caso para saber lo que más favorece al desempeño. El equipo debe estar compuesto por individuos que respondan por la producción de un único producto/servicio, o sea, individuos que contribuyan a un único resultado. Los equipos pequeños facilitan el control de los iguales, si este objetivo fuera importante en un caso concreto. Y también, los equipos pueden ampliarse, con el objetivo de que varias unidades tengan las mismas metas, reforzando la cooperación. De esta forma se espera que la práctica 3, presentada a continuación, genere buenos resultados:

Práctica 3: El modelo debe basarse en metas por equipo y en la premiación por equipo. Hay que estudiar la composición y el tamaño del equipo caso por caso y privilegiar dos criterios: La unicidad de la producción/servicio y el posible control por los iguales.

No es en vano la elaboración de indicadores y metas que orienten los comportamientos deseados. Cada resultado requiere de un conjunto de indicadores que promuevan e incentiven una buena dirección y así evitar el gaming. Esta elaboración demanda estudio, análisis y conocimiento de las políticas públicas. Además, teniendo en cuenta la relación agente x principal, cuanto más información se obtenga acerca del benchmarking, mejor será la efectividad de la relación y la definición de las metas. Sin embargo, no parece posible que las metas difíciles, que conducen a mejores resultados, puedan definirse sin un análisis detallado del desempeño. Por lo tanto es indispensable para la efectividad de modelos de PRP, el establecimiento de una unidad de inteligencia, dotada de alta capacitación y vinculada directamente al principal con el propósito de coordinar el modelo y asesorar a la dirección. De esta forma se espera que la práctica 4, presentada a continuación, genere buenos resultados: 
Práctica 4: Se debe constituir una unidad de inteligencia para negociar metas difíciles, reducir la desigualdad en la información y evitar el gaming.

Parece importante que haya un proceso de establecimiento de la base para el cálculo de la parte variable con el objeto de minimizar el problema de los múltiples principales. Cuanto más formal, sistemático, discutido y negociado el proceso, mejor será el resultado final en términos de efectividad del PRP. Los estudios muestran que la confianza en el modelo, por parte de los empleados, es un factor imprescindible para lograr el éxito. Esta confianza depende de la percepción de estos empleados, si el sistema tiene reglas claras, es justo, transparente y no político. De esta forma se espera que la práctica 5, presentada a continuación, genere buenos resultados:

Práctica 5: Hace falta un proceso formal, sistemático y periódico de contratación de metas cuantitativas que sirva como base del sistema. En este proceso se debe escuchar a los empleados. Se deben establecer unas reglas claras de funcionamiento, divulgarlas y respetarlas. Se debe invertir en la transparencia de las metas y del proceso de evaluación.

Esta teoría muestra claramente que el PRP funciona muy bien con tareas poco interesantes. En este punto del funcionalismo, hay que tener cuidado para que el valor monetario de la premiación no genere grandes presiones de modo que estimule el gaming. El máximo de un 10\%, identificado en las experiencias internacionales, en la remuneración de los funcionarios parece apropiado. De este modo, la práctica 6 parece apropiada:

Práctica 6: El modelado del sistema debe reajustarse en función del tipo de actividad. Un único modelo puede ser ineficaz. Tareas (o funcionarios) con diferentes características demandan modelos de remuneración variable distintos. A nivel operacional, particularmente en sectores que realizan tareas poco interesantes, la implementación del PRP con valores monetarios relativamente bajos es altamente eficaz para mejorar el desempeño.

Al ejecutarse tareas operacionales y poco interesantes, que son objetivos claros para los sistemas de remuneración variable, aparece una cuestión estructural: ¿̨e debe 
implantar el PRP en las otras partes del funcionalismo? No hay una respuesta clara y definitiva a esta pregunta por el momento. Se puede aventurar una hipótesis: Puesto que los efectos positivos y no medibles del PRP están haciendo que este método sea implantado en gobiernos de todo el mundo, se puede deducir que los beneficios derivados son lo suficientemente interesantes para que se asuman los costes ocultos de pérdida de parte de la motivación. Pero no se llega a un consenso. En el caso de las actividades interesantes, otros factores motivacionales como la posibilidad de participar en entrenamientos especializados, recolocación de los funcionarios en acciones estratégicas y la premiación y divulgación de los buenos resultados parecen ser más eficaces ${ }^{14}$. La remuneración variable parece ser un factor motivador muy limitado para este tipo de tareas.

\section{Conclusiones}

El presente trabajo ha mostrado que los sistemas de remuneración variable utilizados en el sector público están fracasando o presentan un éxito limitado. Las explicaciones para este escaso desempeño están compiladas en dos vertientes teóricas. La primera proviene de los trabajos de campo de la economía y se estructura en torno a la teoría de la elección racional y de la relación agente/principal. El argumento se basa en que gran parte de los problemas de la remuneración variable está relacionada a los incentivos modelados incorrectamente y a las características estructurales de la administración pública (Burgess y Ratto, 2003). La segunda vertiente, relacionada con las teorías psicológicas, explica que bajo ciertas circunstancias la remuneración variable no motiva a las personas para tener un mejor desempeño. Según Weibel et al. (2010), en la administración pública los funcionarios presentan por lo general una motivación intrínseca considerable, realizando tareas en función de su sentido del deber, lealtad y placer. Las personas realizan las actividades porque creen en ellas y, al hacerlas, tienen la sensación de que están cumpliendo con su deber. En este contexto, la implementación de sistemas de incentivos monetarios puede generar efectos negativos en la motivación, concretamente en el caso de las tareas interesantes. También se discute una serie de argumentos basados en estudios

14 Argumento del profesor Jobst Fidler (Hertie School of Governance), durante una conferencia en la Fundaçáo Getúlio Vargas-SP titulada Comparative Public Management Reform en $21 / 07 / 2010$. 
empíricos que vislumbraron factores críticos en la implementación, a los cuales los gestores deben prestarles atención: El cumplimiento obligatorio de ciertas etapas en la implementación, la importancia de las metas difíciles, la revelación del fenómeno del gaming y las implicaciones de la cultura brasileńa en el modelado de los esquemas de incentivos.

Se han identificado una serie de prácticas que pueden mejorar los resultados de los sistemas de remuneración variable en el sector público brasileño: 1) la elaboración de una agenda estratégica con indicadores y metas para toda la organización; 2) el establecimiento de resultados cuantitativos, preferencialmente productos/ servicios, como base de la premiación; 3) el uso de metas y premios por equipos; 4) el uso de una unidad de inteligencia contra el gaming; 5) la realización de un proceso formal y periódico de contratación de metas, y 6) la creación de sistemas de incentivos particulares en función del tipo de tareas.

Los argumentos analizados en el presente artículo no dejan duda alguna, es importante mejorar el design y la implementación de los modelos de incentivos. De cualquier forma, el antagonismo entre la opinión pesimista de los académicos y la práctica optimista de los gestores (que están propagando el uso del PRP) puede deberse al diseño de las investigaciones. Los estudios académicos se centran en el éxito o fracaso de los modelos de incentivos en función del análisis de las series históricas de indicadores de desempeño que miden los principales resultados de las organizaciones. Estos estudios son incapaces de apreciar otros aspectos positivos del PRP, como la mejora del desempeño en función de los incentivos en debate en torno de metas y resultados. Los incentivos en debate pueden estimular reflexiones relevantes, alterando eventualmente los productos/servicios y modificar los procesos y estrategias utilizados en el ambiente de trabajo. Un ocasional re direccionamiento de esfuerzos, derivado de este debate, no es captado por los estudios, ya que puede desplazarse el objetivo basado en el desempeño y no ser medido por la misma serie histórica de indicadores. Hacer del desempeño el centro del debate diario de la organización parece tener un importante efecto positivo, sin embargo ha sido poco estudiado hasta el momento. Ésta es una de las lagunas que podrían ser analizadas en futuros estudios. 
Otras dos lagunas del conocimiento podrían resolverse con nuevos estudios. Sin embargo, para la crítica metodológica presentada arriba es fundamental el estudio sobre los resultados que se están obteniendo en las experiencias de PRP en funcionamiento en el gobierno brasileńo. Y, finalmente, las experiencias internacionales nos muestran, de forma contundente, que los distintos modelos y estrategias de implementación son variables importantes para explicar el fracaso o éxito de los sistemas de incentivos. Comprender mejor el impacto de los diferentes modelos y diferentes prácticas de implementación en el contexto nacional es otro paso fundamental para generar una perspectiva más optimista de la implementación de una remuneración variable en el sector público brasileño. 36 ISSN 0717-6759

\section{REFERENCIAS}

Barbosa, Lívia (1996). "Meritoracia à brasileira: o que é desempenho no Brasil?”. Revista do Serviço Público Vol. 120, N³, pp. 58-102.

Behn, Robert (2003). Why measure performance? Different purposes require different measures. Public Administration Review, Vol. 63, № 5, pp. 586-600.

Behn, Robert (2004a). "On the limitations of pay for performance". Public Management ReportVol. $1, N^{\circ} 5$.

Behn, Robert (2004b). "On why public managers should measure outputs”. Public Management Report Vol. 1, № 10.

Behn, Robert (2007). "Danger of using too few measures”. Public Management Report Vol. 5, № 2.

Berheim, B. y Whiston, M. (1986). “Common Agency”. Econometrica Vol. 54, N4, pp. 923-42.

Bowman, James (2010). "The success of failure: the paradox of performance pay". Review of Public Personnel Administration, Vol. 30, $\mathrm{N}^{\circ} 1$, pp. 70-88.

Bilmes, L., E Gould, W. (2009). The people factor: Strengthening America by investing in public service. Washington, DC: Brookings Institution Press.

Burgess, Simon y Ratto, Marisa (2003). "The role of incentives in the public sector: Issues and evidence". Oxford Review of Economic Policy N 19, pp. 285-300. 
Buelens, Marc y Van Der Broeck, Herman (2007). "An analysis of differences in work motivation between public and private organizations". Public Administration Review Vol. 67, N¹, pp. $65-74$.

Dahlström, Carl y Lapuente, Victor (2010). "Explaining cross-country differences in performancerelated pay in the public sector". Journal of Public Administration Research and Theory, $\mathrm{N}^{\circ} 20$, pp. 577-600.

Burgess, S. y Metcalfe, P. (1999). "The use of incentive schemes in the public and private sectors: evidence from british establishments". CMPO Working Paper 99/015.

Eisenhardt, Kathleen M. (1989). Agency theory: an assessment and review. Academy of Management Review, Vo. 14, $\mathrm{N}^{\circ} 1$, pp. 57-74.

Donahue, J. (2008). The warping of government work. Cambridge, MA: Brookings Institution Press.

Gerhart, Barry (2009). “Compensation” en Wilkinson, A.; Bacon, N.; Redman, T. y Snell, S. (editores). Handbook of Human Resource Management. London: SAGE.

Eisenberger, R. y Cameron, J. (1996). "Detrimental effects of reward: reality or myth?” American Psychologist Vol. 51, N¹1, pp. 1153-1166.

François, Patrick (2000). "Public service motivation' as an argument for government provision". Journal of Public Economics Vol. 78, N³, pp. 275-299.

Frank, Sue A., and Gregory Lewis (2004). "Government employees—Working hard or hardly working?” American Review of Public Administration Vol. 34, №1, pp. 36-51.

Hood, Christopher (2006). "Gaming in the target world: the targets approach to managing british public services”. Public Administration Review, Vol. 66, N 4, pp. 515-521.

Grand, Julian. (2006). Motivation, agency, and public policy: of knights and knaves, pawns and queens. Oxford: Oxford University Press.

Kerr, Steven (1975). "On the folly of rewarding A while hoping of B". Academy of Management Journal, Vol. 18, ํ4, pp. 769-83.

Holmström, B. (1982). "Moral harzard in teams". The Bell Journal of Economics, Vol. 13, №2, pp. 324-340.

Ingraham, Patricia (1993). "Of pigs in pokes and policy diffusion-Another look at pay-for-performance”. Public Administration Review Vol. 53, N4, pp. 348-456

Kandel, E. y Lazear, E. (1992). "Peer Pressure and Partnerships". Journal of Political Economics, Vol. 100, N4, pp. 801-817. 
Kellough, J. Edwards, y Haoran Lu. (1993). "The paradox of merit pay in the public sector: Persistence of a problematic procedure”. Review of Public Personnel Administration Vol. 13, $\mathrm{N}^{\circ} 2$, pp. $45-64$

Kelman, Steven (2006). "Improving service delivery performance: organization theory perspectives on central intervention strategies" Journal of Comparative Policy Analysis, Vol. 8, N4, pp. 393-419.

Kerr, Steven (2004). "Editor's introduction: establishing organizational goals and rewards". Academy of Management Executive Vol. 18, N 4, pp. 122-123.

Locke, Edwin A. (2004). "Linking goals to monetary incentives". Academy of Management Executive Vol. 18, N 4, pp. 130-133.

Locke, Edwin A. y Latham, Gary P. (2002). Building a practically useful theory of goal setting and task motivation: a 35-year odyssey. American Psychologist, Vol. 57, $\mathrm{N}^{\circ}$ 9, pp. 705-717.

Marsden, David (2004). "The role of performance related pay in renegotiating the 'effort bargain': the case of the British public service". Industrial and Labor Relations Review Vol. 57, ${ }^{\circ}$ 3, pp. 350-370.

Macdonald, G. y Marx, L. M. (2001). “Adverse specialization”. Journal of Political Economics, Vol. 109, N4, pp. 864-899.

Makinson, J. (2000). Incentives for change: rewarding performance in national government networks. Public Service Productivity Panel

Marsden, David (2010). "The paradox of performance related pay systems: 'why do we keep adopting them in the face of evidence that they fail to motivate?'” en Hood, C.; Margetts, H.; Paradoxes of modernization: unintended consequences of public policy reforms. Oxford, UK: Oxford University Press.

Organization for Economic Co-operation and Development (2005a). Performance-related pay policies for government employees. Paris: OECD Publishing.

Moon, M. (2000). "Organizational commitment revisited in new public management: Motivation, organizational culture, sector, and managerial level”. Public Performance \& Management Review Vol. 24, N², pp. 177-94.

Organization for Economic Co-operation and Development (2005b). Paying for performance: policies for government employees (Policy Brief). Paris: OECD Publishing.

Osterloh, Margit y Frey, Bruno S. (2002). "Does pay for performance really motivate employees?" en Neely, Andy (eds). Business Performance Measurement, Cambridge, Cambridge University Press. 
Pacheco, Regina (2010). "Profissionalização, mérito e proteção da burocracia no Brasil”, en Loureiro, Fernando y Pacheco, Regina (orgs). Burocracia e Política no Brasil: Desafio da Ordem Democrática no Século XXI. Rio de Janeiro: Ed. FGV.

Pacheco, Regina (2009). "Mensuração de desempenho no setor público: os termos de um debate". Actas del Congreso CONSAD.

Perry, James L.; Engbers, Trent A. \& Jun, So Yun (2009). "Back to the future? Performance-related pay, empirical research, and the perils of persistence”. Public Administration Review, Vol. $69, \mathrm{~N}^{\circ} 1$, pp. $1-31$.

Reis Neto, Mário T. y Assis, Luís Otávio M. (2010). “Principais características do sistema de remuneração variável no choque de gestão em Minas Gerais: o Acordo de Resultados e o Prêmio por Produtividade". Gestão \& Regionalidade, Vol. 26, № 76, pp. 75-90.

Trosa, S. (2001). Gestão pública por resultados: quando o Estado se compromete. Rio de Janeiro, Brasília: ENAP.

Weibel A., Rost K., y Osterloh M. (2010). "Pay for performance in the public sector benefits and (hidden) costs". Journal of Public Administration Research and Theory, N²0, pp. 387-412.

Van Dooren, W. (2008). Performance indicators: wolf in sheep's clothing? mimeo

Wilson, James (1989). Bureaucracy: what government agencies do and why they do it. New York: Basic Books. 